\title{
Interacción saludable en la prepa
}

\author{
Jacobet Rosas Yépez y Patricia Moya Grijalva*
}

\section{Resumen}

El presente trabajo es producto de la implementación del curso taller Mi convivencia asertiva en la prepa, el cual se desarrolló de agosto a diciembre de 2017, en el Centro de Estudios Científicos y Tecnológicos del Estado de Sonora (CECyTE) plantel II, en vinculación con el Departamento de Trabajo Social de la Universidad de Sonora. El objetivo medular fue el de otorgar a los estudiantes de nuevo ingreso herramientas y técnicas que favorecen su desarrollo humano y una sana convivencia escolar. Para ello, se ofrecieron ejercicios de autoconocimiento que mejoraron su autoestima y contribuyeron en un óptimo desarrollo escolar; así mismo, se fomentaron hábitos de autonomía a fin de que los estudiantes respondan positivamente ante los factores de riesgo escolar. Se estableció un código de convivencia escolar para mejorar las relaciones entre pares y personal del plantel. Como un aspecto fundamental se trabajó la construcción del proyecto de vida de los participantes, herramienta de realización personal. Este curso taller se fundamentó en conceptos relacionados con el desarrollo humano, procesos de democracia y construcción de la paz, en donde los jóvenes tuvieron la oportunidad de ser escuchados y de forma colectiva construyeron estrategias de convivencia saludable. Los resultados obtenidos fueron favorables y se planea continuar trabajando con los adolescentes de este plantel y replicar el taller en otros centros de estudio de la localidad.

\footnotetext{
* Maestras de tiempo completo de la Licenciatura en Trabajo Social, Universidad de Sonora. Correos: jrosas@sociales.uson.mxy patricia@sociales.uson.mx
} 


\section{Justificación}

Los jóvenes de quince a diecinueve años que cursan la preparatoria, enfrentan cambios biológicos, psicológicos, exigencias sociales y escolares, requieren contar con herramientas y recursos personales, familiares e institucionales que les ayuden a sortear positivamente las dificultades que de ello se genere.

En estudios realizados por instituciones especialistas en la materia, se ha detectado que los problemas más frecuentes que presentan los jóvenes inscritos en preparatorias son los relacionados con los tema de: conflictos en la familia, trastornos alimenticios, trastornos emocionales, entre ellos ansiedad, depresión, riesgos suicidas, baja autoestima, problemas en las relaciones de pareja, abuso de substancias, los de tipo sexual, y los escolares como deserción, reprobación, y violencia escolar. Según datos de la Encuesta Nacional de Exclusión,

Intolerancia y Violencia 2013 (ENEIVEMS), los estudiantes del nivel medio superior de escuelas públicas expresan que se sienten desprotegidos. El $59 \%$ se siente triste, el $44 \%$ se siente solo y el $26 \%$ siente que su vida es un fracaso.

En este sentido la escuela, que es el espacio donde el joven pasa gran parte de su tiempo, debe contribuir al desarrollo integral del estudiante, ya que representa un factor protector en la formación de la identidad y en la planeación del proyecto personal del joven.

Ante este panorama, los planteles requieren de una intervención conjunta, donde otras instituciones se sumen en la atención y prevención de estos fenómenos, de ahí la importancia de la vinculación de la Universidad de Sonora con este sector de la sociedad.

La propuesta de trabajo que se implementó fue en CECyTE, plantel Hermosillo II, con estudiantes de nuevo ingreso a través de actividades que brindaron a los jóvenes herramientas que les permitieron reforzar habilidades sociales, como la autoestima, autonomía, toma de decisiones, la construcción de un código de convivencia saludable y de su proyecto de vida. Así mismo, se reforzaron técnicas de estudio que permitan incidir en los problemas que se presentan y favorezcan su integración en la prepa.

Con este proyecto se coadyuva a mejorar la trayectoria escolar de estos alumnos, evitando su deserción escolar y fomentando el interés por el estudio y por su proyecto de vida, ya que la deserción escolar en este periodo está relacionada con la falta de metas y del proyecto de vida del adolescente, así como con problemas en las relaciones familiares y económicos.

Este curso taller se fundamentó en conceptos relacionados con desarrollo humano y procesos de democracia y construcción de la paz. En este curso taller los jóvenes tuvieron la oportunidad de ser escuchados y de forma colectiva construyeron estrategias de convivencia saludable.

El desarrollo de habilidades sociales en los jóvenes permite fortalecer en ellos la capacidad para identificar y entender sus emociones, sentir $y$ mostrar empatía por los demás, construir y mantener relaciones interpersonales positivas, fijar $y$ alcanzar metas positivas, y tomar decisiones de manera reflexiva $y$ responsable. Todo esto para que puedan enfrentar asertivamente los distintos riesgos a los que están 
expuestos en esta etapa del curso de la vida.

Así pues, este curso taller buscó incidir directamente en el desarrollo integral de los estudiantes, y de manera indirecta en la prevención de conductas de riesgo, tales como violencia, adicciones o embarazo adolescente, que puedan truncar su trayectoria educativa.

\section{Objetivo de la intervención}

Dotar a los estudiantes de nuevo ingreso de herramientas y técnicas que favorezcan su desarrollo humano y una sana convivencia escolar.

\section{Objetivos específicos}

- Mejorar la autoestima a través de ejercicios de autoconocimiento para un óptimo desarrollo escolar

- Fomentar hábitos de autonomía a fin de responder positivamente ante los factores de riesgo escolar

- Establecer un código de convivencia escolar a fin de mejorar las relaciones entre pares y personal del plantel

- Favorecer la construcción del proyecto de vida como herramienta de realización personal.

\section{Fundamentos teóricos}

El curso taller Mi convivencia asertiva en la prepa, se fundamentó particularmente en teorías del desarrollo humano, de construcción de paz y la mediación, lo cual implica enfrentar los conflictos de forma creativa y saludable.

José Antonio Ocampo (2001) define que:

El desarrollo humano no es otra cosa que reconocer la existencia del hombre y de la mujer como seres especiales diferentes a como nos habían acostumbrado a verlos la biología, las corrientes evolucionistas en psicología y en fin un sinnúmero de disciplinas que a través de los años se han atribuido para sí el estudio de la persona y sus diversas potencialidades [...] el desarrollo

humano es como un proceso a través del cual un persona puede llegar efectivamente a construirse como tal, tanto en los aspectos que lo diferencian de los demás y lo hacen singular, como en aquellos que le permiten ser miembro de los colectivos, lo cual le implica su posicionamiento histórico y social.

El desarrollo humano trasciende el pasado y se visualiza hacia el futuro en donde converjan nuevas formas de relacionamientos ante los cambios que se generan en la sociedad, entre ellos:

- El urbanismo creciente de las ciudades que trasforman el sentido del tiempo, pero también las vivencias propias de los espacios.

- La fragmentación social, la pérdida de identidades ante un mundo globalizado.

- La creciente conciencia de grupos minoritarios, género, identidades sexuales entre algunos.

- Las nuevas formas de relacionamiento que implican el uso de las TICS.

- El caso de países donde la política sucumbe ante la corrupción, la impunidad, la sobrevaloración de la arrogancia del dinero fácil, la ausencia de responsabilidades públicas. 
El desarrollo humano en este contexto, busca siempre la justicia, el diálogo, apoyados por la cultura, la educación y las propuestas de los ciudadanos como protagonistas de su desarrollo.

El desarrollo humano no es una condición esencial ya existente a priori, sino un desafío que debe realizarse; de ahí la relevancia de fomentar en los jóvenes su crecimiento como seres humanos, conscientes de su entorno de las necesidades de los otros y de su rol protagónico como sujetos de cambio.

En cuanto a los procesos de democracia y construcción de paz, se puede decir que: la paz debe construirse en la cultura y en la estructura social, no solo en la mente humana. Se deben generar demandas socioeducativas orientadas a desarrollar en los ciudadano un sistemas de valores diferente, un cambio de ideal del hombre materialista al hombre existencial y convivencial, así como la construcción de un paradigma que dé cuenta de la vida y no del dinero.

La escuela adquiere en este escenario relevancia, pues es en ella donde se forma al ciudadano del que se demandan mayores niveles de participación, compromiso, además de una capacidad reflexiva y crítica para ejercer la tarea de la nueva conformación de los espacios relacionales.

Es un hecho de que los espacios para la paz, no pueden ser configurados únicamente desde una sola dimensión de la realidad; también es cierto que la escuela constituye un lugar idóneo de acercamiento para iniciar las acciones de promoción en favor de la edificación de una cultura de paz, pues en el ambiente escolar convergen los diferentes actores de una comunidad: estudiantes, familias, docentes, autoridades, empleados, etc., en quienes se reflejan fenómenos sociales productos de la vida, cultura, valores y creencias de su entorno comunitario.

La escuela es un escenario propenso donde los estudiantes se enfrentan con las contradicciones de la sociedad, con sus amenazas de exclusión, marginación y agresión, por un lado, y con las oportunidades de aprender maneras de enfrentarlas, por otro. Algunas escuelas pueden convertirse en escenarios de violencia en los que se puede aprender, la intolerancia, el chantaje y la corrupción. Del mismo modo, puede aprenderse en ellas la solidaridad, el respeto a la diversidad o la honradez. Las competencias para ser ciudadanos se aprenden. Y la mejor manera de hacerlo es consolidar una cultura democrática en el sistema escolar. Es decir, en la escuela se aprende a ser ciudadano mediante el ejercicio práctico de la democracia.

La medición escolar es otro elemento que contribuye a resolver los conflictos en la comunidad escolar de forma pacífica. El potenciar en los jóvenes las habilidades sociales les permite ser mejores personas e interactuar de forma saludable con sus pares y docentes, resolviendo las diferencias interpersonales de forma pacífica. Así mismo, se pretende que los involucrados en los conflictos puedan desarrollar habilidades de comunicación, asertividad (poder expresar las opiniones y los puntos de vista, sean correctos o no), identificar emociones, empatía, escucha activa.

\section{Contextualización y reconstrucción de la práctica}

CECyTE es una institución de nivel medio superior creada bajo la directriz de la Dirección General de Educación Tecnológica Industrial (DGETI) y adscrita a la Dirección General de CECyTE Sonora. En su modalidad de bachillerato tecnológico, busca facilitar el acceso a la educación media superior y a la preparación técnica, 
principalmente a jóvenes de comunidades rurales y semiurbanas, así como promover el interés por el desarrollo social y cultural de su entorno, atendiendo a jóvenes de catorce a dieciocho años.

EI CECyTE II Alto Valle, fue el plantel específico en el que se implementó el curso taller Mi convivencia asertiva en la prepa. Se sitúa al norte de la ciudad de Hermosillo, en avenida del Parque final s/n esquina con prolongación Yáñez, colonia Alto Valle. Los jóvenes que acuden a este plantel son de diversos estratos sociales, mayor mente de clase baja. Esta institución fue creada en el sexenio del licenciado Manlio Fabio Beltrones Rivera, el 31 de julio de 1996, bajo el artículo 23 del reglamento interior de la Secretaría de Educación Pública, como un organismo público descentralizado, con personalidad jurídica y patrimonios propios, para atender la demanda educativa a nivel medio superior.

El proyecto fue implementado por estudiantes del sexto semestre y dirigido por docentes de la Licenciatura en Trabajo Social de la Universidad de Sonora, ante la necesidad de dar respuesta a la demanda de CECyTE de vincular nuestras acciones en beneficio de los estudiantes.

Se mantuvo coordinación con la orientadora educativa del plantel CECyTE II de Hermosillo, Sonora. Se trabajó durante el semestre escolar 2017-2 con un grupo de treinta estudiantes de primer semestre (grupo $P)$, a fin de proporcionarle elementos que contribuyan a su permanencia, integración y culminación de sus estudios favoreciendo una convivencia pacífica en la preparatoria.

Cabe señalar que esta práctica coadyuva y complementa los objetivos del programa del gobierno mexicano "Construye T", dirigido y financiado por la Secretaría de
Educación Pública (SEP), a través de la Subsecretaría de Educación Media Superior (SEMS), e implementado con el apoyo del Programa de las Naciones Unidas para el Desarrollo (PNUD). Su objetivo es fortalecer las capacidades de la escuela para desarrollar habilidades socioemocionales en las y los jóvenes, y así mejorar el ambiente escolar.

\section{Descripción de la práctica}

El desarrollo de las actividades del curso taller se fundó en el modelo centrado en el participante. Se le dio voz a este en la construcción de su aprendizaje y se fomentó la cooperación entre pares.

El modelo centrado en el participante:

Permite un aprendizaje más profundo y permanente, propicia el desarrollo de habilidades, actitudes y del pensamiento crítico; el proceso didáctico se centra en la actividad del participante. El instructor lo apoya directamente. El proceso de enseñanza se subordina a que el aprendizaje se desarrolle de la mejor manera. Desarrollan las habilidades del participante para aprender a aprender. Pasa a segundo plano la labor informativa, siendo lo prioritario la labor formativa. Exige un trabajo previo del participante aun cuando se trate de técnicas de enseñanza en grupo, se intenta personalizar el proceso de enseñanza y llegar al alumno concreto, individual. Estimulan la socialización, pues ayudan a que el participante comprenda y respete a los otros, e interactúe con ellos. Se centra en el trabajo colaborativo. Ayudan a ligar los conocimientos a la práctica. Procuran un aprendizaje significativo (Griorgis y Minjangos, 2014, p. 19).

El curso taller tuvo como
característica trabajar mediante la
exposición de elementos teóricos por


parte del instructor, para que posteriormente el estudiante los aplicará en ejercicios prácticos y vivenciales.

Las técnicas utilizadas fueron:

- Integración y participación grupal

- Análisis, reflexión de situaciones y casos

- Resolución de problemas

- Construcción de proyectos

Los contenidos temáticos del curso taller y los resultados obtenidos en cada uno de ellos fueron:

1. Autoestima: jóvenes con un mejor autoconocimiento

2. Código de convivencia: jóvenes con mejor convivencia escolar

3. Autonomía: jóvenes con mejor capacidad de responder ante los retos de la vida

4. Técnicas de estudio: jóvenes dotados de técnicas de estudio

5. Proyecto de vida: jóvenes con metas a corto y mediano plazo definidas

Estos contenidos se desarrollaron del 31 de agosto al 26 de octubre, repartidos en una sesión por semana de dos horas. Se obtuvo un total de dieciocho horas presenciales y las dos horas restantes, fueron repartidas en tareas de quince minutos vivenciales por cada sesión, sumando un total de veinte horas.

El material didáctico de cada sesión fue elaborado por los instructores, basados en material bibliográfico sobre el tema y el uso de las nuevas tecnologías, Así mismo, se sustentaron en los materiales didácticos proporcionados en el Diplomado semipresencial con mediación virtual en: Procesos de Construcción de Paz y Democracia con Participación de Niños,
Niñas y Jóvenes, en Hermosillo, Sonora, el cual fue orientado a fortalecer las dinámicas de relación entre los diferentes actores sociales que permitan consolidar una vida humana digna, así como una convivencia pacífica que promueva la participación infantil y juvenil en los procesos intergeneracionales en la construcción del camino hacia la paz y la democracia en contextos de alta conflictividad social. Este diplomado fue impartido por la Universidad de Manizales Colombia.

Los estudiantes evaluaron positivamente el taller a través de una encuesta y comentarios grupales, externando que recomendarían el taller a otros jóvenes. También que les gustaría que se abordaran temas relacionados con la sexualidad y relaciones familiares en talleres similares.

\section{Interpretación crítica}

En términos generales se puede decir que se lograron los objetivos al brindar a los estudiantes herramientas para mejorar su autoestima, lo que potenció su desarrollo escolar. Se fortalecieron elementos en la toma de decisiones que les van a permitir actuar con autonomía y responder positivamente ante los factores de riesgo escolar. Se reforzaron los valores para la convivencia escolar lo que favoreció relaciones saludables entre pares y personal del plantel. Así mismo, se proporcionaron elementos para la construcción de un proyecto de vida que permitirá organizar sus tareas tanto escolares, familiares, laborales para alcanzar las metas que como sujetos se propongan.

Durante la ejecución del proyecto se observó, en el comportamiento grupal, un cambio en las relaciones entre pares. También durante el desarrollo de las 
actividades fue aumentando la participación, respeto e integración del grupo, lo que permitió el logro de los objetivos.

Los estudiantes evaluaron favorablemente, tanto el contenido como la forma de trabajo implementada en el curso taller. Se manifestó que fue de utilidad lo abordado y que recomendarían a otros el mismo.

Cabe mencionar que se estableció enlace con el $\mathrm{H}$. Ayuntamiento de Hermosillo para implementar el programa "Hazlo mejor", a fin de continuar fortaleciendo los valores en la comunidad estudiantil del plantel.

La institución manifestó interés y apertura para que el personal del Departamento de Trabajo Social, replique este proyecto con otros grupos de estudiantes.

\section{Conclusión}

Este tipo de experiencias es necesario replicarlas en los estudiantes de otros centros educativos, en virtud del impacto positivo que tiene en las relaciones entre pares y docentes. Continuar con enfoques de desarrollo humano y procesos de construcción de paz, en los cursos talleres con los jóvenes, potencializa un panorama esperanzador en las alternativas de solución a los conflictos y problemas que aquejan a esta población, permeando las esferas de la familia y la comunidad.

La sugerencia es que se retome en la formación de los jóvenes, el camino de los sentidos y significados, poner el acento en la construcción de un sujeto más seguro y confiado en sí mismo, propiciando mejores condiciones dentro del aula, en la organización escolar, en las familias y en el barrio o ambientes en que los adolescentes se desenvuelven, abriendo espacios de participación activa y rescatando los valores y principios de la convivencia democrática en todas las instancias en las que transita el estudiante.

\section{Prospectiva}

Se prende continuar replicando este tipo de talleres, adecuando los temas a las necesidades que cada plantel y grupo, procurando que las estudiantes de trabajo social implementen y pongan en práctica sus conocimientos teóricos y metodológicos, y los principios éticos profesionales.

\section{Bibliografía}

AENOR. (2008). Técnicas de Estudio. España: Vértice.

André, C. y Lelord, F. (2009). La Autoestima: gustarse a sí mismo para mejor vivir con los demás. ( $5^{\mathrm{a}}$ ed.). Barcelona: Kairos.

Beltrán, G.R. (s/f). Imaginar lo humano: algunas consideraciones alrededor del Desarrollo Humano. Ensayo.

Bravo, N. (1997). Valores humanos por la senda de una ética cotidiana. ( $6^{\mathrm{a}}$ ed.). Santiago de Chile: Ril Editores.

Cardona, A. (2000). Formación de valores: Teoría, reflexiones $y$ respuestas. México: Grijalbo.

Subsecretaría de Educación Media Superior. (2013). Encuesta Nacional de Exclusión, Intolerancia y Violencia en Escuelas Públicas de Educación Media Superior (ENEIVEMS). México.

Flores, A., Morlán, S., Pérez, M., Nava, A. y López, J. (2004). Yo mi proyecto de vida. México: Panorama.

Flores, M. y Díaz, R. (2002). Asertividad: una alternativa para el óptimo manejo de las relaciones interpersonales. México: Universidad Autónoma de Yucatán. 
Foderaro, A., \& Schmidt, D. (2012). Tu decisión para adolescentes. Guía de sentido común para tomar mejores decisiones. E.U.A.: Author House.

Gil Ospina, A. (2006). Algunas consideraciones alrededor del desarrollo humano. Revista Zona, 1.

Giorgis, R. y Mijangos, J. (2014). Androgogía modelos, métodos y técnicas. Recuperado el 15 de marzo de 2016 de: https://issuu.com/rinagiorgis/docs/proy ecto_final_-josu_y_rina_1

Goleman, D. (2006). La inteligencia emocional. México: Vergara.

González, R. (2005). Guía para la formación y crecimiento personal del joven. México: Universidad de Colima.

Güell Barceló, M. (2006). ¿Por qué he dicho blanco si quería decir negro? Técnicas asertivas para el profesorado y formadores. Barcelona: GRAÓ.

Hare, B. (2003). Sea asertivo la habilidad directiva clave para comunicar eficazmente. Barcelona: Gestión 2000.

Jiménez, J. y González, J. (2004). Método para desarrollar hábitos y técnicas de estudio: bachillerato y universidad. España: La tierra hoy.

Moll, S. (2015). 7 Técnicas asertivas para afrontar con éxito una discusión. Recuperado el 16 de marzo de 2017 de: http://justificaturespuesta.com/tecn icas-asertivas-para-afrontar-discusion/

Ocampo, J.A. (2001). Retomar la agenda del desarrollo. Ensayo presentado en el panel de la reunión anual de la Asociación Estadounidense de Economía. Nueva Orleans: E.U.

Quiroga, B. (2014). Los adolescentes y la toma de decisiones. Recuperado el 6 de junio de 2016 de: http://universoup.es/9/hoja-de- ruta/los-adolescentes-y-la-toma-dedecisiones/

Subsecretaría de Educación Media Superior. (2017). Programa Construye T 2014-2018 Fortalecer las capacidades de la escuela para promover el desarrollo integral de los jóvenes. Recuperado el día 15 de marzo de 2017 de: http://www.construye-t.org.mx/

Salazar Castilla, M., Loaiza de la Pava, J.A. (2014). Curso-taller procesos de democracia y construcción de paz con niños, niñas y jóvenes. México: Universidad de Sonora. 